\title{
Water Absorption Behaviour and Mechanical Performance of Pineapple Leaf Fibre Reinforced Polylactic Acid Composites
}

\author{
E. H. Agung ${ }^{*}$, M. H. M. Hamdan ${ }^{2}$, J. P. Siregar ${ }^{2}$, D. Bachtiar ${ }^{2}$, C. Tezara ${ }^{3}$ \\ and J. Jamiluddin ${ }^{2}$
}

\author{
${ }^{1}$ Engineering Department, Faculty of Engineering, Universitas Malahayati, Jl. Pramuka \\ No. 27, Kemiling, Bandar Lampung, 35153, Indonesia. \\ *Email: efriyo@abulyatama.ac.id \\ Phone: +62721271117 Fax: +62721271119 \\ ${ }^{2}$ Structural Materials and Degradation Focus Group, Faculty of Mechanical \\ Engineering, Universiti Malaysia Pahang, 26600 Pekan, Pahang, Malaysia \\ ${ }^{3}$ Mechanical Engineering Department, Faculty of Engineering \& Quantity Surveying, \\ INTI International University, Persiaran Perdana BBN, 71800 Nilai, Negeri Sembilan, \\ Malaysia.
}

\begin{abstract}
Fast-growing scientific work is focusing on alternative sources to replace modern synthetic fibre materials due to the adverse effects caused by petroleum-based materials. Natural fibre possesses high potential as a replacement for synthetic fibre and petroleumbased products. These materials are not only greener and environmental-friendly, but also safe for human health. As such, this study investigated the influence of compatibilising agent of maleated anhydride polyethylene (MAPE) on mechanical performance of pineapple leaf fibre (PALF) reinforced polylactic acid (PLA). The raw materials, such as PALF, PLA, and MAPE, were mixed by using a hot roller mixer machine and hot compression moulding at $190^{\circ} \mathrm{C}$. The specimens were then tested for water absorption and flexibility. The specimens were submerged in water for $0,7,14$, and 21 days. Three types of tests were conducted, namely water absorption, tensile, and flexural assessments. The results of water absorption, tensile, and flexural tests for the untreated PALF composite (UPALF) and treated PLAF composite (TPALF) were recorded and explained. As a conclusion, composite materials based on hydrophilic natural fibre may reduce the tensile and flexural properties of the composite.
\end{abstract}

Keywords: Mechanical properties; pineapple leaf fibre; polylactic acid; water absorption; maleated polyethyelene.

\section{INTRODUCTION}

Recently, academia, scientists, and researchers have been focusing on natural fibre reinforced composite partly to preserve the earth from further pollution due to the use of petroleum-based materials. The petroleum-based composite consumes enormous energy and causes problems at the end of its life cycle [1]. To date, the viable option to solve the problems related to petroleum-based composite is by implementing materials that are derived from natural resources. The natural fibre is well-known for its own advantages, such as renewability, biodegradability, high availability, high corrosion resistance, lightweight, and cost-effectiveness, when compared to synthetic fibre counterparts [2, 3]. Nevertheless, the main weaknesses of natural fibre are low thermal resistance and 
vulnerable to water absorption due to the existence of polar group, which is root for hydrophilic nature of natural fibre [3, 4]. Despite that, a number of factors could drive the water absorption of natural fibre, such as fibre content, immersion temperature, exposure of area to water, void content, fibre orientation, and permeability of fibres $[5,6]$.

The marriage of natural fibre with biodegradable matrix bio-polymer results in excellent green material that is compatible with the environment. Polylactic acid (PLA), which originates from natural source, such as corn and sugar, is a biodegradable polymer material that has excellent mechanical properties, such as high strength and modulus [7]. It degrades upon exposure to the environment in a short period, which is about two years, when compared to petroleum plastics that take an average of 8 years [8,9]. Previous researchers had looked into the natural fibre reinforced PLA composite [10]. For instance, bamboo, vetiver grass and coconut fibres were used as reinforcement fibre for the composite $[8,11]$. Flexible epoxy resins were used for surface treatment on the fibres. The epoxy resin with $1 \mathrm{wt}$ \% of reinforcement was dissolved in acetone to reduce the viscosity prior to surface treatment process. After the fibres had been treated, they were left to dry at room temperature for one whole day. The fibres were prepared in 10, 20, 30, and $40 \%$ by weight [12]. Many researchers have performed numerous analyses upon the PLA with varied natural elements. Nevertheless, the study of pineapple leaf fibre (PALF) and PLA is relatively new and limited.

PALF is a natural fibre that has potential to serve as an alternative to synthetic fibre due to its economic and renewable nature. PALF is seen to have superior mechanical properties because of its high content of $\alpha$-cellulose. Furthermore, PALF is suitable to serve as reinforcing fibre in composite because of its excellent qualities compared to other natural fibres $[13,14]$. The PALF, as reinforcement material, has high content of cellulose and exhibits outstanding mechanical performance, especially in terms of tensile, flexural, and impact, which is desirable for making high quality polymer composite. The PALF fibre has an average density of $1530 \mathrm{~kg} / \mathrm{m}^{3}$ and tensile strength of approximately 290.61 $\mathrm{MPa}$ [2]. The chemical composition of PALF fibre has about 70\%-82\% holocellulose, $5 \%-12 \%$ lignin, and $1.1 \%$ ash [15]. Similar to other natural fibre, PALF is also susceptible to moisture absorption.

Surface modification is necessary to overcome the hydrophilic nature of natural fibre. Surface modification will eventually reduce water absorption, besides enhancing interfacial adhesion of fibre and matrix polymer $[8,16]$. Many options for treatment is available to perform surface modification on natural fibre, such as alkaline, compatibilising agent, permanganate, peroxide, and benzoylation [17, 18]. One favourite treatment among researchers is the compatibilising agent of maleic anhydride due to ease of processing and cost-effectiveness [19]. The most outstanding compatibilising agents are maleated anhydride of polyethylene (MAPE) and maleated anhydride polypropylene.

However, literature on the performance of PALF reinforced PLA under excessive moisture condition is still limited to date especially with influence of compatibilising agent which is MAPE. Therefore, this study determined the dimensional stability of PALF reinforced PLA composite under the influence of excessive moisture condition. Moreover, the behaviour of water penetration into the composite was assessed by looking into its diffusivity, sorption coefficient, and permeability coefficient. In addition, the investigation was carried out with two different conditions, namely dry and wet conditions. 


\section{METHODOLOGY}

\section{Materials}

This experiment employed three materials: biopolymer PLA as the matrix, PALF as the reinforcement material, and MAPE as the compatibilising agent. NatureWorks, USA supplied the thermoplastic biopolymer of PLA (IngeoTM Biopolymer 2003D) used in the study. The PLA with pellet shape had a density of $1.24 \mathrm{~g} / \mathrm{cm}^{3}$ and melt flow index of 6 $\mathrm{g} / \mathrm{min}$. The PALF was obtained from West Java, Indonesia. The PALF fibre used in this study had an exact density of $1.46 \mathrm{~g} / \mathrm{cm}^{3}$ Lastly, the MAPE (OREVAC®18302N) was obtained from Tazdiq Engineering, Kuala Lumpur. The MAPE pellet had a density of $0.912 \mathrm{~g} / \mathrm{cm}^{3}$ and melt flow index of $1.2 \mathrm{~g} / 10 \mathrm{~min}$.

\section{Fabrication of Composites}

In order to prepare the composites, PALF was washed with clean water to remove any foreign material or residue on the surface that may affect the bonding strength. After washing, the fibres were dried under sunlight to remove the moisture. Then, the PALF was ground to acquire sizes that ranged between 2 and $4 \mathrm{~mm}$. The vacuum oven was used to dry the PALF at $80{ }^{\circ} \mathrm{C}$ for almost 24 hours. Next, the PALF was sealed in closed containers. The PLA, MAPE and untreated PALF (UPALF) / treated PALF (TPALF) were mixed based on the formulation described in Table 1 .

Table 1. Formulation for PALF reinforced PLA composite

\begin{tabular}{lccc}
\hline Group name & $\begin{array}{c}\text { Fibre weight } \\
\text { content }(\%)\end{array}$ & $\begin{array}{c}\text { Resin weight } \\
\text { content }(\%)\end{array}$ & $\begin{array}{c}\text { MAPE weight } \\
\text { content }(\%)\end{array}$ \\
\hline UPALF & 10 & 90 & 0 \\
\hline TPALF & 10 & 88 & 2 \\
\hline
\end{tabular}

The mixing process of PALF and PLA was performed by using a roll mixer machine at $190{ }^{\circ} \mathrm{C}$ and a constant speed of $50 \mathrm{rpm}$. Upon completion, the composite compound was cooled at room temperature. The process was continued with a different type of formulation. Then, the crusher machine was used to turn the PLA-PALF mixture into a pellet. After that, the composite pellet was stored in a container for the next process.

The composite plate with a dimension of $20 \mathrm{~cm} \times 20 \mathrm{~cm} \times 0.3 \mathrm{~cm}$ was prepared via hot compression moulding. The temperature and pressure used for the compression moulding were $190^{\circ} \mathrm{C}$ and $15 \mathrm{MPa}$, respectively. The finished product of PALF reinforced PLA composite was cut via table-saw into the dimensions as proposed in ASTM D638 and ASTM D790.

\section{Water Absorption Analysis}

The water absorption testing was conducted according to ASTM D570-98 [20]. Distilled water at $25{ }^{\circ} \mathrm{C}$ was used in this testing. Five different time periods were set in this experiment: $0,1,7,14$, and 21 days. The weight of the specimen was measured by using a high precision balance based on the period and recorded accordingly. The experiment was halted when all the samples attained their saturation points, as they remained constant 
in weight. Eq. (1) and Eq. (2) were employed to measure the thickness of swelling and the rate of water absorption.

$$
\% \text { Thickness Swelling }=\left(\frac{\mathrm{t}_{2}-\mathrm{t}_{1}}{\mathrm{t}_{1}}\right) \times 100
$$

As depicted in Eq. (1), $t_{1}$ represents thickness of dry sample and $t_{2}$ is thickness of wet sample at the set time. The percentage of water absorption is presented in Eq. (2).

$$
\% \text { Water absorption }=\left(\frac{\mathrm{W}_{2}-\mathrm{w}_{1}}{\mathrm{w}_{2}}\right) \times 100
$$

In Eq. 2, $\mathrm{w}_{1}$ and $\mathrm{w}_{2}$ refer to samples before and after immersion in the distilled water at certain period. The diffusivity, permeability coefficient, sorption coefficient, and permeability coefficient parameters were determined by using the formula portrayed in Eq. (3), Eq. (4), and Eq. (5).

$$
\begin{aligned}
& \text { Diffusion coefficient }(\mathrm{D})=\pi\left(\frac{\mathrm{t}^{2} \mathrm{~m}^{2}}{16 \mathrm{w}_{\infty}}\right) \\
& \text { Sorption coefficient }(S)=\frac{M_{\infty}}{M_{t}}
\end{aligned}
$$$$
\text { Permeability coefficient }(\mathrm{P})=\mathrm{D} \times \mathrm{S}
$$

From Eq. (3), as described above, $t$ represents initial thickness of the composite in $\mathrm{mm}$, and $m$ constant is slope of initial linear portion of $\frac{\mathrm{w}_{\mathrm{t}}}{\mathrm{w}_{\infty}}$ versus $\sqrt{\mathrm{t}}$. The $w_{\infty}$ is weight percentage of the sample at an infinite time. Besides, $\mathrm{M}_{\infty}$ denotes percentage of mass at infinite time, while $M_{t}$ is mass sample at initial time. The experimental data of the PALF composite were fitted in Eq. (6).

$$
\log \left(\frac{w_{t}}{w_{\infty}}\right)=\log \mathrm{m}+\mathrm{n} \log \mathrm{t}
$$

In Eq. (6), $\mathrm{n}$ is indication for diffusion mechanism. In the case of $\mathrm{n}=0.5$, the diffusion is Fickian. In the case of non-Fickian or anomalous diffusion, the value of $n$ is between 0.5 and 1 .

\section{Tensile Test}

The tensile test on the PALF composite was carried out by adhering to ASTM 638 type 4 [21]. The PALF composite had an overall length of $115 \mathrm{~mm}$ and thickness of $3 \mathrm{~mm}$. The tensile testing was conducted by using a Universal Testing Machine (UTM) (Model 3369, Instron, Norwood, MA). The crosshead speed for the tensile test was $1 \mathrm{~mm} / \mathrm{min}$. A total of seven specimens were prepared for the test. The data retrieved from PALF composite analysis were calculated to obtain the average for each group of specimens. 


\section{Flexural Test}

The flexural test in this experiment was performed by adhering to ASTM D790 [22]. The UTM machine employed for tensile testing was used with minor adjustment. The crosshead speed was fixed at $2 \mathrm{~mm} / \mathrm{min}$. The support span of the sample was set up with a ratio of 1:16. A $30 \mathrm{kN}$ load was used. The flexural test was repeated for seven times. The values of the flexural strength and modulus were analysed to acquire their averages.

\section{Analysis of Surface Fracture}

The fracture sample of tensile testing was carried out for the study of surface morphology via SEM (Evo® 50 Series, Carl Zeiss AG, Oberkochen, Germany). Before that, the sample was sputtered with titanium for about 10 minutes to ensure electric charging during the examination.

\section{RESULTS AND DISCUSSION}

\section{Water Absorption on PALF/PLA Composites}

The specimen underwent a process of water absorption by submerging the sample into water for the following days: $0,1,7,14$, and 21 days. Each test consisted of 7 specimens. The effect of water absorption was determined from four different physical properties of a specimen, such as its weight, thickness, width and length. The measurements of width, length and thickness on UPALF and TPALF composites for $0,1,7,14$, and 21 immersion days are recorded in Tables 2 and 3, respectively.

Table 2. Change in dimension and weight of UPALF composites.

\begin{tabular}{ccccccccc}
\hline & \multicolumn{8}{c}{ Average change of dimension } \\
Day & $\begin{array}{c}\text { Width } \\
(\mathrm{mm})\end{array}$ & $\begin{array}{c}\text { Dimensional } \\
\text { increase }(\%)\end{array}$ & $\begin{array}{c}\text { Length } \\
(\mathrm{mm})\end{array}$ & $\begin{array}{c}\text { Dimensional } \\
\text { increase }(\%)\end{array}$ & $\begin{array}{c}\text { Thickness } \\
(\mathrm{mm})\end{array}$ & $\begin{array}{c}\text { Thickness } \\
\text { swelling } \\
(\%)\end{array}$ & $\begin{array}{c}\text { Weight } \\
(\mathrm{g})\end{array}$ & $\begin{array}{c}\text { Water } \\
\text { absorption } \\
(\%)\end{array}$ \\
\hline 0 & 12.86 & - & 127.25 & - & 2.45 & - & 4.90 & - \\
1 & 12.88 & 0.15 & 127.52 & 0.21 & 2.52 & 2.86 & 5.29 & 7.96 \\
7 & 12.89 & 0.23 & 127.62 & 0.29 & 2.69 & 9.80 & 5.66 & 15.51 \\
14 & 12.94 & 0.62 & 127.76 & 0.40 & 2.72 & 11.02 & 5.84 & 19.18 \\
21 & 13.00 & 1.09 & 127.77 & 0.40 & 2.73 & 11.43 & 5.84 & 19.18 \\
\hline
\end{tabular}

Table 3. Change in dimension and weight of TPALF composites.

\begin{tabular}{ccccccccc}
\hline & \multicolumn{7}{c}{ Average change of dimension } \\
Day & $\begin{array}{c}\text { Width } \\
(\mathrm{mm})\end{array}$ & $\begin{array}{c}\text { Dimensional } \\
\text { increase }(\%)\end{array}$ & $\begin{array}{c}\text { Length } \\
(\mathrm{mm})\end{array}$ & $\begin{array}{c}\text { Dimensional } \\
\text { increase }(\%)\end{array}$ & $\begin{array}{c}\text { Thickness } \\
(\mathrm{mm})\end{array}$ & $\begin{array}{c}\text { Thickness } \\
\text { swelling } \\
(\%)\end{array}$ & $\begin{array}{c}\text { Weight } \\
(\mathrm{g})\end{array}$ & $\begin{array}{c}\text { Water } \\
\text { absorption } \\
(\%)\end{array}$ \\
\hline 0 & 13.00 & - & 127.37 & - & 2.72 & - & 5.26 & - \\
1 & 13.03 & 0.23 & 127.44 & 0.06 & 2.74 & 0.74 & 5.27 & 2.92 \\
7 & 13.19 & 1.46 & 127.46 & 0.07 & 2.82 & 3.68 & 5.43 & 6.05 \\
14 & 13.22 & 1.69 & 127.63 & 0.20 & 2.84 & 4.41 & 5.50 & 7.42 \\
21 & 13.24 & 1.85 & 127.63 & 0.20 & 2.84 & 4.41 & 5.50 & 7.42 \\
\hline
\end{tabular}

The dimensions for UPALF and TPALF composites can be compared from Tables 1 and 2. The length and width of both UPALF and TPALF composites had no significant 
changes. However, analysis on thickness swelling present that the TPALF composites have more dimensional stability compared UPALF composite.

The water absorption result also presents that TPALF has better resistance in water absorption compared to UPALF composites. The results for 21-day immersion show that TPALF composites have $7.42 \%$ of water absorption compared to UPALF composites which result in $19.18 \%$. Although TPALF composites show an enhancement in water absorption, the result is still higher compared with the previous study. The finding from Espert et al. (2004) presented that the composites consisted of $10 \%$ of combination cellulose fibre (sisal, coir and luffa sponge) reinforced polypropylene (PP) which had $6 \%$ of water absorption for 21-day immersion [23]. It is due to the water absorption in natural fibre composites being highly influenced by the type of polymer matrix, form of reinforcement material and different water parameter (acidity or water condition, and temperature) [24].

Figure 1 shows that the UPALF and the TPALF composites recorded $11 \%$ and $4 \%$ thickness of swelling. The thickness of swelling had a similar pattern with water absorption. Figure 1 indicates that the thickness swelling of PALF/PLA composites increases with longer immersion time. The water absorption affects the dimensional stability by a build-up of moisture in fibre cell wall, which causes the swelling of the fibre $[13,25]$. Other factors that contribute to the high thickness swelling of PALF composite are the high content of lignocellulosic material and the capillary action [25]. Both factors seem to promote water intake on the composite material when soaked in distilled water. The dimensions of the specimen also alter due to the nature of PALF. The change in physical properties may affect the performance of PALF composite in tensile and flexural testing. However, the introduction of MAPE reduces the thickness of swelling for UPALF by $61 \%$.

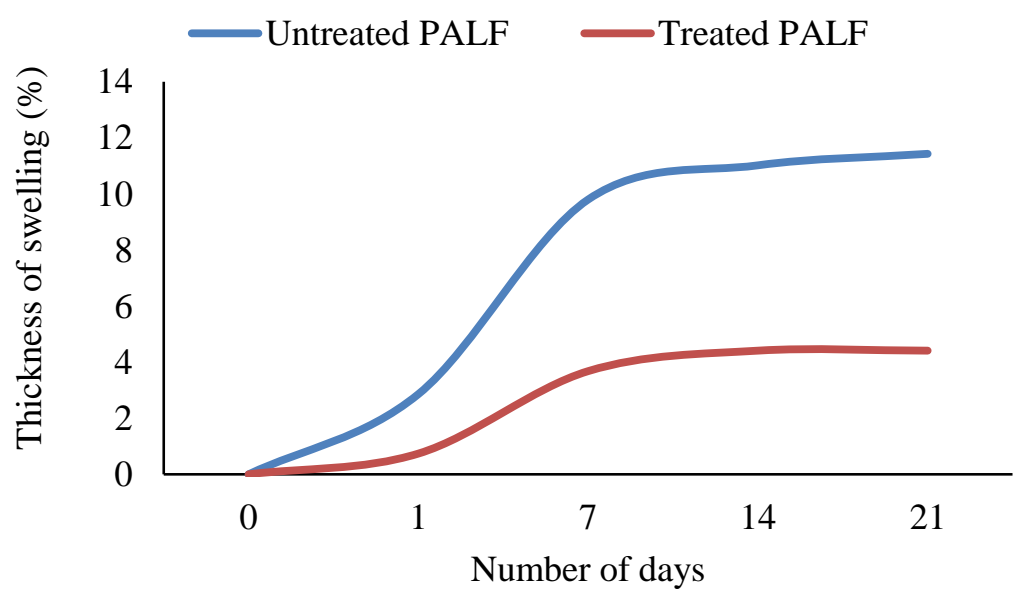

Figure 1. Water absorption of UPALF and TPALF against the square root of time.

Figure 2 illustrates the water absorption characteristics of UPALF and TPALF composites. The UPALF composite yielded about $19 \%$ percentage of water absorption, while the TPALF yielded about 7\%, which is lower when compared to UPALF composite by $56.7 \%$. Both UPALF and TPALF composites absorbed a significant amount of water in the early stage of immersion, but the absorption turned slow after hitting saturation point. Danladi and Shu'aib (2014) [26] who investigated PALF with HDPE composite also revealed similar finding and suggested that the high absorption of water by PALF was motivated by its rich cellulose content [27]. 
The surface modification via MAPE had successfully reduced the moisture absorption of the composite. This finding displays agreement with a prior work that utilised the MAPE [28]. The TPALF fibre might absorb less moisture that directly improved its adhesion to the polymer matrix. MAPE generated bonding between PALF and matrix polymer, thus resulting in a change in its mechanical performance and the minimised sensitivity towards water.

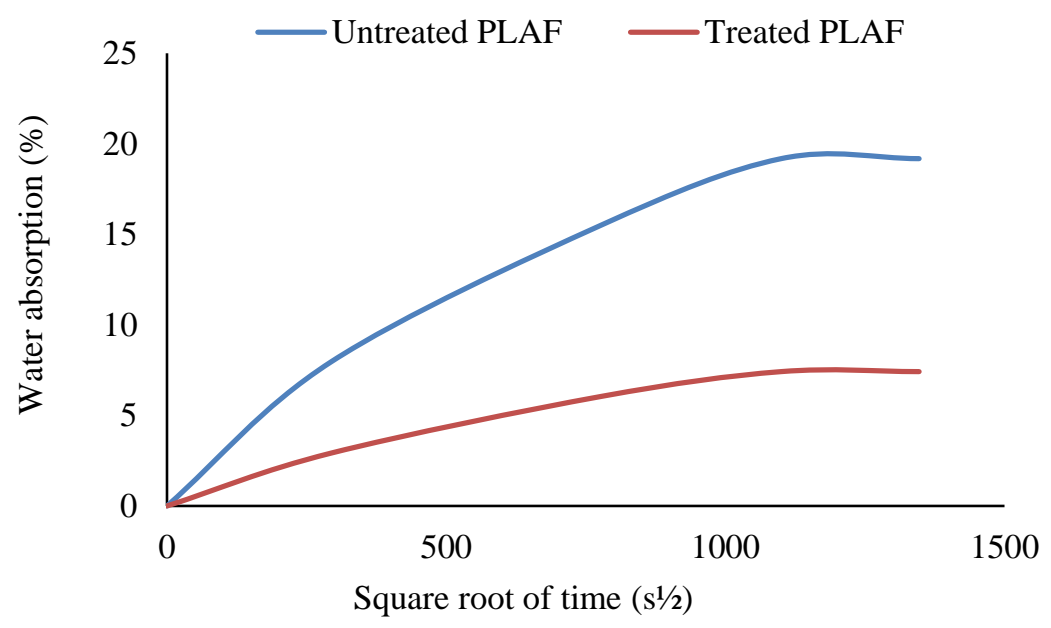

Figure 2. Water absorption of UPALF and TPALF against the square root of time.

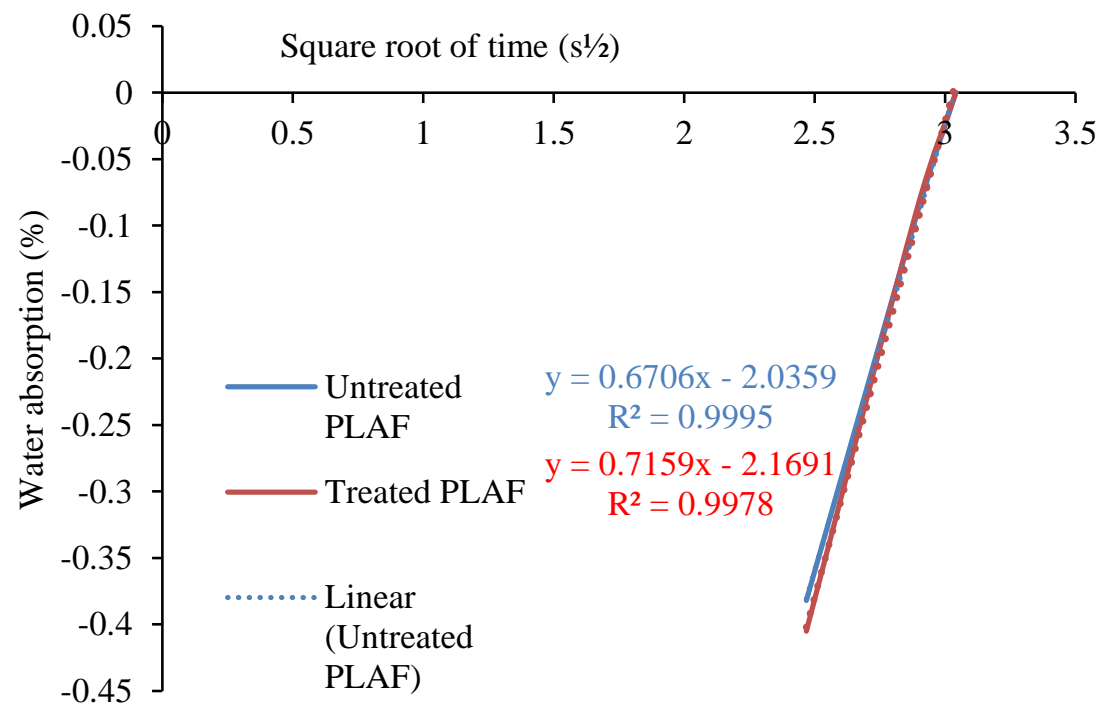

Figure 3. Curve fitting for diffusion kinetic for determining value ' $m$ ' and ' $n$ ' for UPALF and TPALF

The value of ' $\mathrm{m}$ ' for UPALF and TPALF were recorded to be around 0.00920 and 0.00538 , respectively. Meanwhile, the values of ' $n$ ' for UPALF and TPALF were 0.67 and 0.71 . The values of ' $n$ ' indicated that both composites experienced non-Fickian $(0.5<$ ' $n$ ' $<1$ ) diffusion. In the case of non-Fickian, the diffusion and relaxation are almost identical. Therefore, the trend of water absorption seem to never reach the equilibrium 
[29]. The non-Fickian behaviour could be inherited from the existence of the micro crack on the surface and inside the PALF composite encourage the water transport mechanism becoming more active [30].

The values for all sorption coefficient, diffusion coefficient, and permeability coefficient are summarised in Table 4. The TPALF composite had the lowest sorption coefficient, when compared to that of the UPALF. The diffusion coefficient obtained on the experiment are in agreement as obtained by the researchers on the other polymer fibre reinforced composite [31, 32]. Table 3 shows that the TPALF composite has the lowest sorption coefficient, when compared to that of UPALF. This verifies that the TPALF composite displays better adhesion between fibres and matrix than that of UPALF fibre. However, the diffusion coefficient and the permeability coefficient of TPALF composite are significantly better. The value of sorption coefficient highly depends on the fraction of the PALF content, as well as the void content on the composite sample.

Table 4. Change in dimension and weight of TPALF composites.

\begin{tabular}{lcc}
\hline & UPALF & TPALF \\
\hline Sorption coefficient, S & 1.191 & 1.046 \\
Diffusion coefficient, D $\left(\mathrm{mm}^{2} / \mathrm{s}\right)$ & $2.72 \times 10^{-07}$ & $7.64 \times 10^{-07}$ \\
Permeability coefficient, P $\left(\mathrm{mm}^{2} / \mathrm{s}\right)$ & $3.24 \times 10^{-07}$ & $7.99 \times 10^{-07}$ \\
\hline
\end{tabular}

\section{Tensile Strength of PALF/PLA Composites}

The results of tensile strength and tensile modulus for UPALF and TPALF composites are presented in Figures 4 and 5, respectively. Tensile strength and tensile modulus for the UPALF composite before being immersed into water at room temperature were recorded at 35.6 MPa and 1.854 GPa. The value of UPALF obtained on the experiment are more significant compared to the result obtained by Munawar et al. (2015) [32] on different types of PALF (Moris Gajah, Jasopine, Maspine, and N36) reinforced PLA even though they possess similar fibre loading.

Figure 4 shows that the tensile strength of UPALF decreased drastically by about $52 \%$ when immersed on the first day. After 21 days, the UPALF composite lost about $81.9 \%$ from the original value. The UPALF also became stiffer when immersed into water for an extended period. The value of Young modulus for UPALF had dropped by about $49 \%$ at the end of the immersion time. The tensile properties of TPALF composite also deteriorated when exposed to water. The reduction in tensile performance when exposed to excessive water absorption are similar and common in many cases as shown by previous research work [33]. Nonetheless, the percentage drop of the tensile performance depended on many factors. As highlighted by Rashidi et al. (2009) [34], the composite with fibre reinforced containing higher cellulose suffered most on the mechanical performance due to the higher percentage of moisture uptake. Nonetheless, the volume fraction of PALF utilised on the study is slightly equal for both cases of UPALF and TPALF. The difference between the UPALF and TPALF composite could be inherited from the interfacial adhesion of PALF and PLA. When exposed to water, the interfacial between PALF and PLA weakened, which led to more degradation of the composite sample [6]. The water that penetrated into the composite disrupted the fibre-matrix bonding [35]. As a result of the addition of MAPE, the effect of water absorption on the TPALF composite was minimum than that of the UPLAF composite. The tensile strength of TPLAF composite only dropped by about $50 \%$ on the first day, reaching $73 \%$ after 21 
days. The highest loss of tensile modulus of TPALF composite after 21 days was about $30.95 \%$.

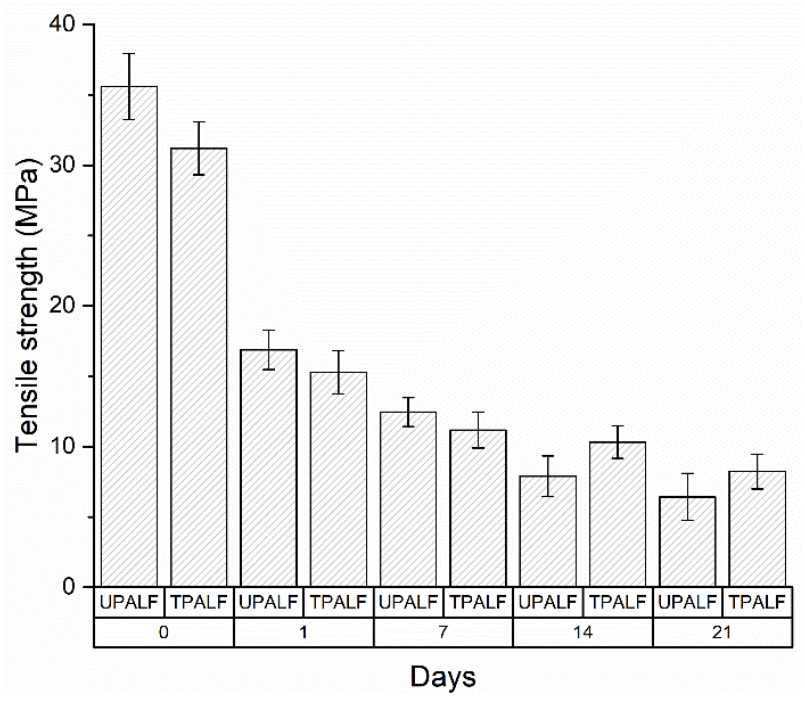

Figure 4. Tensile strength of UPALF and TPALF composites under the influence of water absorption.

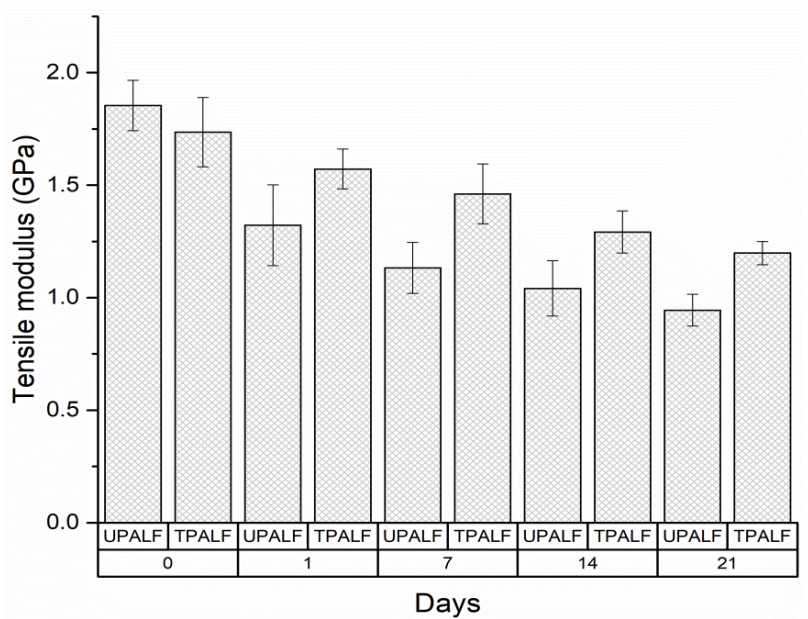

Figure 5. Tensile modulus of UPALF and TPALF composites under the influence of water absorption.

The TPALF recovered the losses in tensile performance, which suggests enhancement of effectiveness with the addition of MAPE. Without MAPE, the PALF composite would be vigorously attacked by water absorption, thus becoming a weaker material.

\section{Flexural Strength of PALF/PLA Composites}

Figures 6 and 7 show the results obtained from flexural testing for UPALF and TPALF composites. Figure 6 presents that the UPALF composite had a flexural stress value at about 46.66 MPa, while for TPALF composite the value was 75.27 MPa. Besides, the flexural modulus for UPALF was about $2.63 \mathrm{GPa}$. After being immersed in distilled water 
for $1,7,14$, and 21 days, the value of the flexural strength seemed to have decreased. The UPALF loss was only about $5 \%$ of the original strength after a day of immersion and the loss continued up to $38 \%$ at the end of the immersion time. The highest flexural strength obtained was $75.27 \mathrm{MPa}$, while the flexural modulus was about $3.45 \mathrm{GPa}$. Therefore, the alkaline treatment did improve the UPALF composite by about $60 \%$ and $31 \%$ for flexural strength and flexural modulus, respectively. The TPALF composite dropped by about $3 \%$ and continued to decrease until 29\% from the original strength after 21 days of immersion time. A similar pattern of decreasing flexural strength and flexural modulus was also observed for flexural modulus.

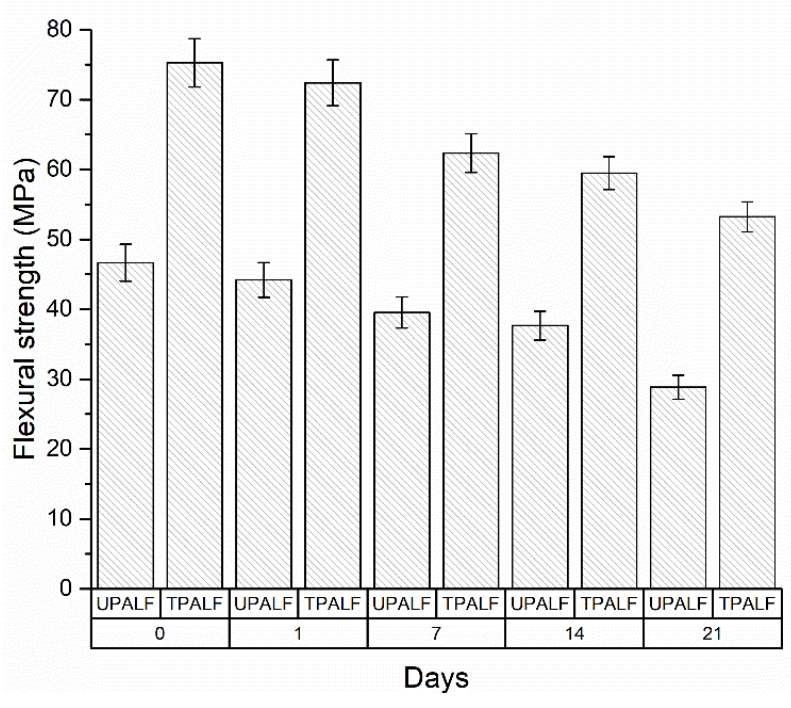

Figure 6. Flexural strength of UPALF and TPALF composite under the influence of water absorption

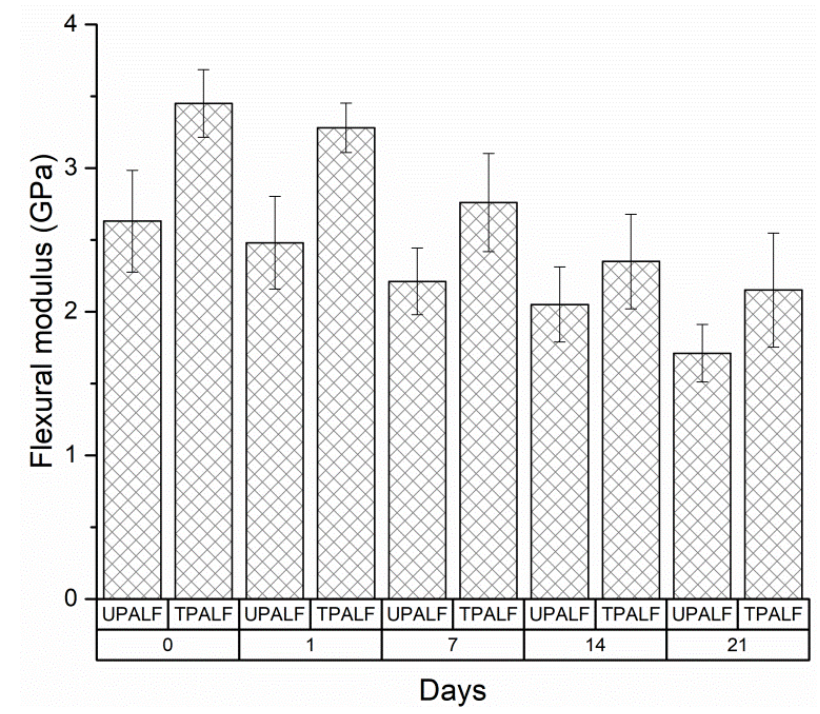

Figure 7. Flexural modulus of UPALF and TPALF composite under the influence of water absorption.

PLA is relatively unstable when exposed to high moisture environment, thus influenced by immersion time and temperature. As a result, water absorption causes 
rigidity loss. The drop in the flexural and flexural modulus is due to the water molecules that rapidly enter the composite as a result of capillary action. As a consequence, the bonding between the fibre and the matrix is weakened at the initial stage. After reaching the saturation point, the crack and the void on the composite were filled with water that acts as plasticiser, and it can be in favour of tensile strength. The reason for TPALF composite to have higher flexural strength and modulus than UPALF composite is due to the presence of void on the composite interface eliminated by MAPE. The roles of MAPE on flexural properties are similar to those in tensile properties. MAPE improves flexural properties by limiting water absorption and improving the interfacial adhesion on PALF and PLA [6].

\section{Surface Morphology of PALF Composite}

Figure 8 shows the surface morphology fracture sample obtained from tensile testing performed upon UPALF and TPALF composites. Figure 8 shows that no fibre pull-out was found on the composite surface, which indicated the strong bonding and interfacial adhesion between PALF and PLA composite. Nevertheless, as shown in Figures 8(a) and 8(b), the void content on the surface is observed. Void in the composite is highly related to both mechanical performance and absorption capability. Therefore, the composite with less void volume usually exerts good mechanical performance and high resistance for water absorption [35]. As illustrated in Figure 8(a), the size of a void in the UPALF is bigger than that in the TPALF composite. The more significant appearance of a void in the UPALF reduced its tensile performance and flexibility. Therefore, MAPE treatment on the composite was able to minimise the void in terms of volume and size. The existence of void is due to the entrapped air and moisture inside the fibre bundle of PALF. As higher volume of air or moisture is trapped inside the composite, the composite will display poor mechanical performance. The images of sample fracture for both UPALF and TPALF appeared to be consistent with the results of tensile and flexural performance.

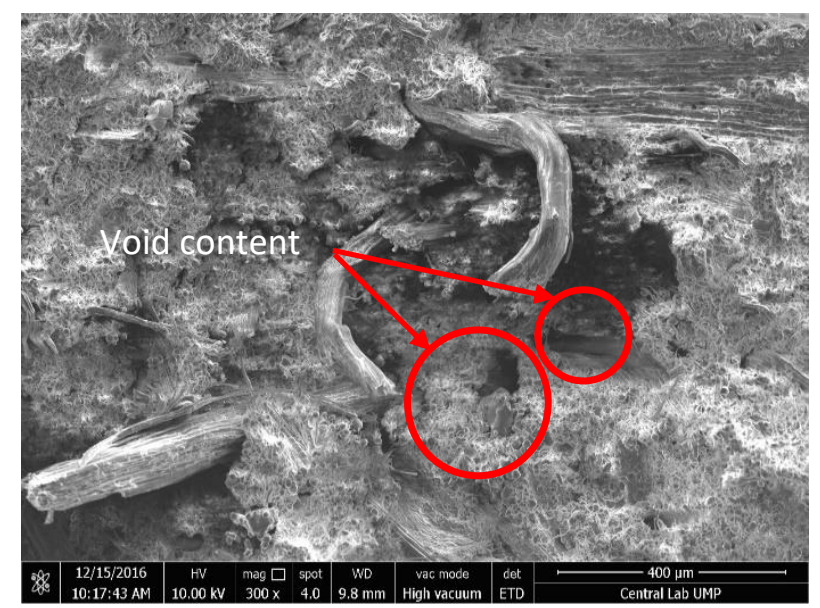

(a) 


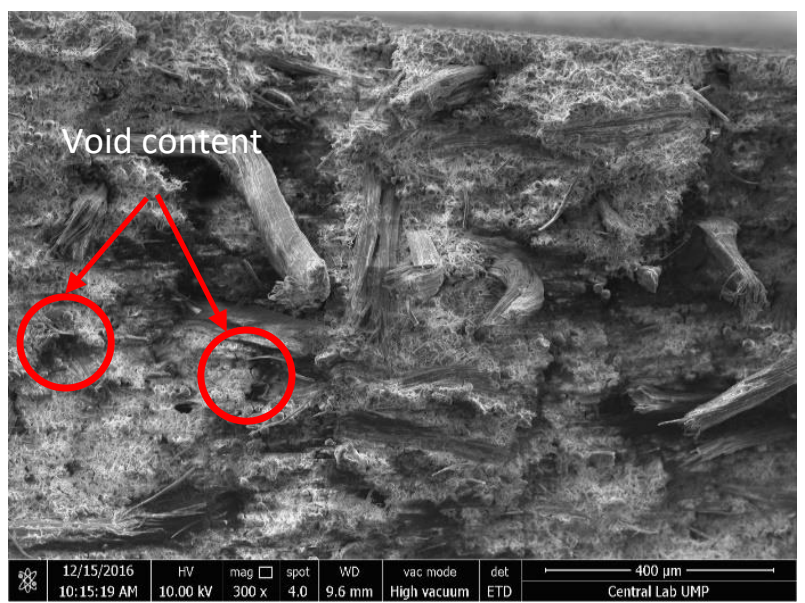

(b)

Figure 8. Fracture image for PALF reinforced PLA: (a) UPALF (b) TPALF.

\section{CONCLUSION}

This study was performed by combining agricultural residue that consists of PALF and biopolymer of PLA. The exploitation of PALF and PLA opened a new type of bio composite that is harmless to the environment throughout its life cycle, from production, usage, and disposal. Generally, the PALF reinforced PLA is susceptible when exposed to the outdoor condition especially in excessive water due to the biodegradable properties of the PLA polymer. In the experiment, the influence of water absorption on the dimensional stability and mechanical performance was discovered. Moreover, the effect of compatibilising agent of MAPE was studied and compared to the untreated PALF reinforced PLA. Several key results were obtained:

i. Low water absorption and changes in dimension had been recorded for the TPALF composite compared to the UPALF. Both specimens follow the non-Fickian behavior.

ii. The tensile properties of UPALF and TPALF deteriorate when immersed in distilled water for a short period.

iii. From the flexural testing, degradation of bonding between PLAF and PLA is the main reason for the drop in flexural performance.

iv. The utilisation of MAPE successfully improved the tensile and flexural performance of the PALF reinforced PLA composite about $27.83 \%$ and $84.5 \%$ on the $21^{\text {st }}$ day.

v. SEM observation indicated that the size and distribution of the void content are minimised when MAPE was introduced to the PALF reinforced composite. Elimination of void content reduced the water penetrated via the capillary action. The finding can be summarised that MAPE successfully provides resistance to the water by promoting good adhesion between PALF and PLA composite. Future work should focus on other types of chemical treatment to reduce water absorption in PALF.

\section{ACKNOWLEDGEMENT}

The authors would like to thank Universitas Malahayati and Universiti Malaysia Pahang for the provision of exceptional laboratory facilities. 


\section{REFERENCES}

[1] Sudhakara P, Jagadeesh D, Wang Y, Prasad C, Devi AK, Balakrishnan G, Kim B, Song J. Fabrication of Borassus fruit lignocellulose fiber/PP composites and comparison with jute, sisal and coir fibers. Carbohydrate polymers. 2013; 98(1):1002-1010.

[2] Anbukarasi K, Kalaiselvam S. Study of effect of fibre volume and dimension on mechanical, thermal, and water absorption behaviour of luffa reinforced epoxy composites. Materials \& Design. 2015; 66:321-330.

[3] Misnon MI, Islam MM, Epaarachchi JA, Wang H, Lau KT. Woven hemp fabric reinforced vinyl ester composite: effect of water absorption on the mechanical properties degradation. International Journal of Advances in Science, Engineering and Technology. 2016; 4(3):96-101.

[4] Kushwaha PK, Kumar R. Influence of chemical treatments on the mechanical and water absorption properties of bamboo fiber composites. Journal of Reinforced Plastics and Composites. 2011; 30(1):73-85.

[5] Daramola OO, Adediran AA, Adewuyi BO, Adewole O. Mechanical properties and water absorption behaviour of treated pineapple leaf fibre reinforced polyester matrix composites. Leonardo Journal of Sciences. 2017; 16(30):15-30.

[6] Tissandier C, Zhang Y, Rodrigue D. Effect of fibre and coupling agent contents on water absorption and flexural modulus of wood fibre polyethylene composites. in AIP Conference Proceedings. 2014. AIP.

[7] Al-Mobarak T, Gafur MA, Mina MF. Preparation and characterization of raw and chemically modified sponge-gourd fiber reinforced polylactic acid biocomposites. Materials Sciences and Applications. 2018; 9(02):281.

[8] Jamiluddin J, Siregar JP, Sulaiman A, Jalal K, Tezara C. Study on properties of tapioca resin polymer. International Journal of Automotive and Mechanical Engineering. 2016; 13:3178-3189.

[9] Jamiluddin J, Siregar JP, Tezara C, Hamdan M, Sapuan SM. Characterisation of cassava biopolymers and the determination of their optimum processing temperatures. Plastics, Rubber and Composites. 2018; 1-11.

[10] Dimzoski B, Bogoeva-Gaceva G, Srebrenkoska V, Avella M, Gentile G, Errico M. Preparation and characterization of poly (lactic acid)/rice hulls based biodegradable composites. Journal of Polymer Engineering. 2008; 28(6-7):369383.

[11] Sujaritjun W, Uawongsuwan P, Pivsa-Art W, Hamada H. Mechanical property of surface modified natural fiber reinforced PLA biocomposites. Energy Procedia. 2013; 34:664-672.

[12] Siakeng R, Jawaid M, Ariffin H, Sapuan SM, Asim M, Saba N. Natural fiber reinforced polylactic acid composites: A review. Polymer Composites.

[13] Gupta M, Singh R. PLA-coated sisal fibre-reinforced polyester composite: Water absorption, static and dynamic mechanical properties. Journal of Composite Materials. 2018; 0021998318780227.

[14] Lopattananon N, Panawarangkul K, Sahakaro K, Ellis B. Performance of pineapple leaf fiber-natural rubber composites: the effect of fiber surface treatments. Journal of Applied Polymer Science. 2006; 102(2):1974-1984.

[15] Jaafar J, Siregar JP, Oumer AN, Hamdan MHM, Tezara C, Salit MS. Experimental Investigation on Performance of Short Pineapple Leaf Fiber 
Reinforced Tapioca Biopolymer Composites. BioResources. 2018; 13(3):63416355.

[16] Asim M, Jawaid M, Abdan K, Ishak M. Dimensional stability of pineapple leaf fibre reinforced phenolic composites. in AIP Conference Proceedings. 2017. AIP Publishing.

[17] Asim M, Abdan K, Jawaid M, Nasir M, Dashtizadeh Z, Ishak M, Hoque ME. A review on pineapple leaves fibre and its composites. International Journal of Polymer Science. 2015; 2015:1-16.

[18] Siregar J, Sapuan SM, Rahman M, Zaman H. The effect of alkali treatment on the mechanical properties of short pineapple leaf fibre (PALF) reinforced high impact polystyrene (HIPS) composites. Journal of Food, Agriculture \& Environment. 2010; 8(2):1103-1108.

[19] Jaafar J, Siregar JP, Piah MBM, Cionita T, Adnan S, Rihayat T. Influence of Selected Treatment on Tensile Properties of Short Pineapple Leaf Fiber Reinforced Tapioca Resin Biopolymer Composites. Journal of Polymers and the Environment. 2018; 26(11):4271-4281.

[20] ASTM, ASTM D570 - 98 Standard Test Method for Water Absorption of Plastics. 1998, ASTM International: West Conshohocken, PA.

[21] ASTM, ASTM D 638-03 Standard Test Method for Tensile Properties of Plastics. 2003, ASTM International: West Conshohocken, PA.

[22] ASTM, ASTM D790-02 Standard Test Method for Flexural Properties of Unreinforced and Reinforced Plastics and Electrical Insulating Materials. 2002, ASTM International: West Conshohocken, PA.

[23] Espert A, Vilaplana F, Karlsson S. Comparison of water absorption in natural cellulosic fibres from wood and one-year crops in polypropylene composites and its influence on their mechanical properties. Composites Part A: Applied science and manufacturing. 2004; 35(11):1267-1276.

[24] Nair MM, Shetty N. Effect of Distilled and Sea Water Absorption on Mechanical Behaviour of Short Coir Fibre Epoxy Composite/Sawdust Filler. Pertanika Journal of Science \& Technology. 2018; 26(1): 261-282.

[25] Atiqah A, Jawaid M, M Ishak, Sapuan SM. Moisture absorption and thickness swelling behaviour of sugar palm fibre reinforced thermoplastic polyurethane. Procedia engineering. 2017; 184:581-586.

[26] Jawaid M, Khalil HA, Khanam PN, Bakar AA. Hybrid composites made from oil palm empty fruit bunches/jute fibres: Water absorption, thickness swelling and density behaviours. Journal of Polymers and the Environment. 2011; 19(1):106109.

[27] Danladi A, Shu'aib J. Fabrication and properties of pineapple fibre/high density polyethylene composites. American Journal of Materials Science. 2014; 4(3):139143.

[28] Husseinsyah S, Azmin AN and Ismail H. Effect of maleic anhydride-graftedpolyethylene (MAPE) and silane on properties of recycled polyethylene/chitosan biocomposites. Polymer-Plastics Technology and Engineering. 2013; 52(2):168174.

[29] Zamri MH, Akil HM, Bakar AA, Ishak ZAM, Cheng LW. Effect of water absorption on pultruded jute/glass fiber-reinforced unsaturated polyester hybrid composites. Journal of composite materials. 2012; 46(1):51-61. 
[30] Girisha C, Sanjeevamurthy G, Srinivas G. Sisal/coconut coir natural fibers-epoxy composites: Water absorption and mechanical properties. International Journal Engineering \& Technology Innovation. 2012; 2:166-170.

[31] Leman Z, Sapuan SM, Saifol A, Maleque M, Ahmad M. Moisture absorption behavior of sugar palm fiber reinforced epoxy composites. Materials \& Design. 2008; 29(8):1666-1670.

[32] Uma Devi L, Joseph K, Manikandan Nair K, Thomas S. Ageing studies of pineapple leaf fiber-reinforced polyester composites. Journal of applied polymer science. 2004; 94(2):503-510.

[33] Munawar RF, Jamil NH, Shahril MK, Rahim SMSA, Abidin MZZ, Azam MA, Lau K-T. Development of Green Composite: Pineapple Leaf Fibers (PALF) Reinforced Polylactide (PLA). Applied Mechanics \& Materials. 2015; 761.

[34] Rashdi A, Sapuan SM, Ahmad M, Khalina A. Water absorption and tensile properties of soil buried kenaf fibre reinforced unsaturated polyester composites (KFRUPC). Journal of Food, Agriculture \& Environment. 2009.

[35] Graupner N, Herrmann AS, Müssig J. Natural and man-made cellulose fibrereinforced poly(lactic acid) (PLA) composites: An overview about mechanical characteristics and application areas. Composites Part A: Applied Science and Manufacturing. 2009; 40(6):810-821.

[36] Alomayri T, Assaedi H, Shaikh FUA, Low IM. Effect of water absorption on the mechanical properties of cotton fabric-reinforced geopolymer composites. Journal of Asian Ceramic Societies. 2014; 2(3):223-230.

[37] Hwang CL, Huynh TP. Investigation into the use of unground rice husk ash to produce eco-friendly construction bricks. Construction and Building Materials. 2015; 93:335-341. 